\title{
Angiolymphoid Hyperplasia with Eosinophilia (ALHE): A Diagnostic Dilemma
}

\section{Puri V, Khare $\mathrm{N}^{*}$ and Deshpande $\mathrm{ON}$ \\ Department of Plastic Surgery, Seth GS Medical College \& KEM Hospital, India}

*Corresponding author: Dr Nishant Khare, Deptt. Of Plastic Surgery, Seth GS Medical College \& KEM Hospital, Mumbai - 12, India, Tel: 08224055822; Email: drnishantkhare@gmail.com

\section{Case Report \\ Volume 2 Issue 1}

Received Date: January 12, 2017

Published Date: February 01, 2017

DOI: $10.23880 /$ cdoaj16000111

\section{Abstract}

Angiolymphoid hyperplasia with eosinophilia (ALHE) is a benign vaso proliferative disease of undetermined origin. It presents with painless, vascular nodules in the dermal and subcutaneous tissues of the head and neck, particularly around the ear. It is thought to arise due to a atopic hypersensitivity reaction. The lesion is often confused with other possible causes of similar lesions like mesenchymal tumour of the vessels, nerves or muscles in the head and neck, Kimura's disease etc. Complete excision and follow-up is the treatment of choice which is associated with a $33 \%$ recurrence rate. We are presenting a case of ALHE which presented mimicking a vascular malformation in the pretemporal region in a 26 year old male.

Keywords: ALHE; Mesenchymal tumour; Hemangiomas; Kimura's disease; Lesions

\section{Introduction}

Angiolymphoid hyperplasia with eosinophilia (ALHE) is a benign vasoproliferative disease of undetermined origin. It is characterized by the presence of nodular pseudo-tumors corresponding microscopically to a vascular proliferation within an inflammatory infiltrate made up of lymphocytes, macrophages, and eosinophils. It is a rare entity and closely resembles other more common lesions like Hemangiomas, Mesenchymal tumors, Kimura's disease etc.

\section{Case Report}

A 26 year old male patient presented with a slow growing, painless swelling over the right pre-auricular region (Figure 1). The patient had noticed the lesion 6 months prior to seeking treatment. The swelling slowly grew in size and caused cosmetic embarrassment to the patient. There was no history of trauma, or infectious lesion of the scalp. Patient had been asymptomatic and without any history of fever or pain. There had been no subcutaneous swelling anywhere else. An attempted surgery at another centre was abandoned suspecting a vascular malformation.

On presentation there was a $3 X 2 \mathrm{~cm}$ globular, firm, mobile swelling seen over the right pre-auricular region. The swelling was non tender, non compressible, non fluctuant and non expansile. Another globular lesion $1 \mathrm{X} 0.6 \mathrm{~cm}$ in size was seen just adjacent to it. A multiplanar 3D CT Angiography was done using $1 \mathrm{~mm}$ collimation. Enhancing vessels were seen adjacent and posterior to the lesion encased by the tumour. The provisional diagnosis was that of a pseudo-tumor/hemangioma/giant cell arteritis. Ultrasound showed a well defined echo poor lesion with minimal posterior enhancement suggestive of hemangioma/neuroma. 
The FNAC was suggestive of a pigmented spindle cell lesion. Hematological parameters were normal except for the raised eosinophil count. Patient underwent excisional biopsy of the lesion. On exploration, the lesion was seen in the subcutaneous plane. The swellings were closely adherent to the superficial temporal artery. The patient had an uneventful postoperative recovery. The histopathological examination revealed a smooth encapsulated soft to firm nodule brownish in colour on cut section. On microscopy, an un-encapsulated nodule composed of numerous capillary sized blood vessels with predominantly plump endothelial cells, a large number of eosinophils and dense lymphoid infiltration in a fibromyxoid stroma. Lymphoid follicles were not seen. The biopsy was confirmative of ALHE.

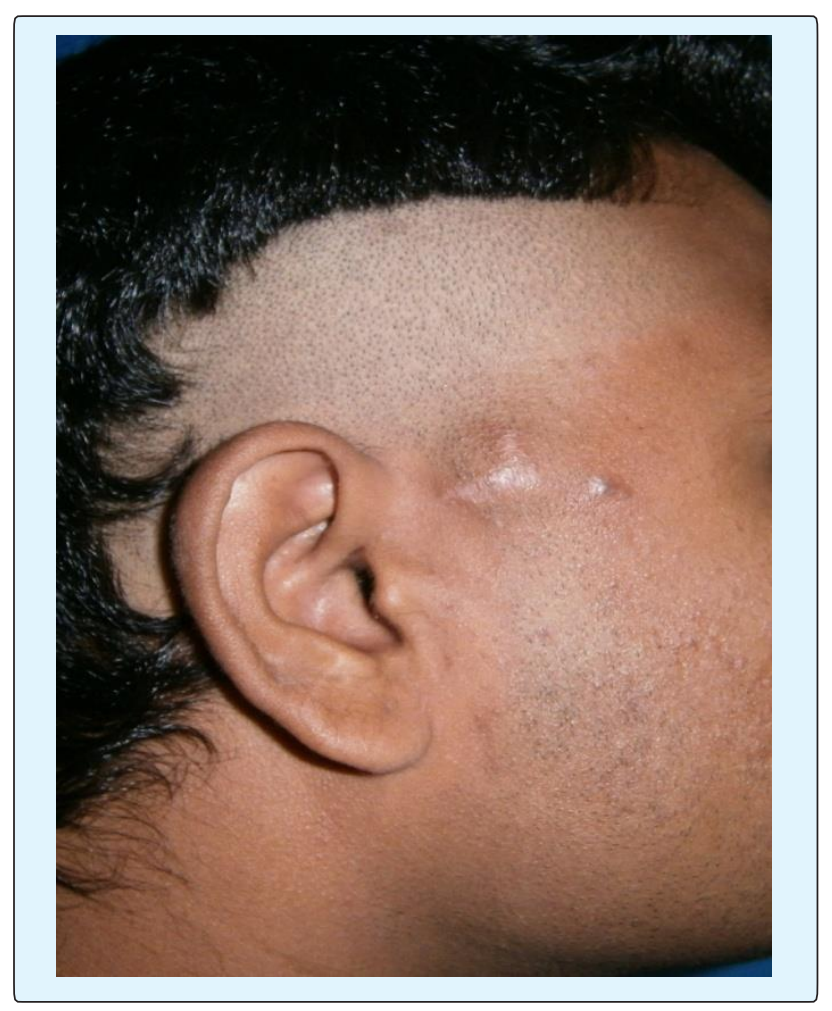

\section{Discussion}

Angiolymphoid hyperplasia with eosinophilia is an uncommon, benign, reactive vaso-proliferative disease, presenting with painless, vascular nodules in the dermal and subcutaneous tissues of the head and neck, particularly around the ear [1]. ALHE has also been reported in the scalp [2], lip [3], tongue [4], orbits [5], conjunctiva, muscles and bone [2-6]. ALHE is more common in females; however, a male predominance has been noted in selected Asian studies. It presents most commonly in patients aged 20-50 years, with a mean onset of 30-33 years. The condition is uncommon; it is rare in elderly patients and in the non-Asian paediatric population [7]. The present case is a 26 year old male patient which correlates with some Asian studies.

ALHE was first described in Wells and Whimster [8], the typical lesion of ALHE are isolated or small numbers of red-brown papules in the head and neck region. The lesion ranges between 1 to $10 \mathrm{~cm}$ in size [8]. ALHE is thought to arise due to a atopic hypersensitivity reaction. However, its exact origin is disputed and neoplasia, infection and trauma have been suggested as other possible etiologies [9]. The lesion is often confused with other possible causes of similar lesions like mesenchymal tumour of the vessels, nerves or muscles in the head and neck, namely, neurilemmoma, leiomyoma, hemangioma, and fibrosarcoma [10]. The present case also presented with a $3 \mathrm{X} 2 \mathrm{~cm}$ lesion in the preauricular region which is the most common location described for this rare lesion.

Several treatment options have been suggested for ALHE. The uses of intra-lesional injections of isotretinoin, glucocorticoids, interferon alpha-2a, cytotoxic agents, and irradiation therapy have been described in the literature [9]. However, complete excision and follow-up is the treatment of choice [2]. It is also the most frequent treatment as the diagnosis of ALHE is often made on postoperative histopathological examination. Excision is associated with a $33 \%$ recurrence rate [9]. The present patient had undergone a complete excision of the lesion and is recurrence free at 3 month follow up. The patient will be followed up for a longer duration of time before labeling him as recurrence free.

Clinically the condition resembles Kimura's disease, though the lesions tend to be more superficial and lymph node involvement is less common [11]. Microscopically they are clearly different: Kimura's disease is characterized by the presence of numerous lymphoid follicles with distinct germinal centres and an abundance of dendritic cells. IgE has been identified in the germinal centres of Kimura's disease but not ALHE [12]. ALHE is characterised by a distinctive infiltrate, composed of lymphocytes, eosinophils, and plasma cells. The condition is confirmed by the histopathological presence of abnormally proliferating, plump endothelial cells often in the vicinity of muscular arteries [12].

The present case had all the cardinal clinical and pathological features of ALHE and yet the pre-operative diagnosis was not made. As a matter of fact, the initial histopathological diagnosis was also that of Kimura's disease and it was only after review of relevant literature 
that the diagnosis was revised to ALHE. This is due to the fact that owing to rarity of the lesion the index of suspicion is low. We therefore recommend that ALHE should be considered in the differential diagnosis of such lesions.

\section{Conclusion}

ALHE is a rare but distinct entity mimicking Kimura's Disease and other more common vascular lesions. It should be considered in the differential diagnosis of such lesions in the head and neck region.

\section{Reference}

1. Zaraa I, Mlika M, Chouk S, Chelly I, Mokni M, et al. (2011) Angiolymphoid hyperplasia with eosinophilia: a study of 7 cases. Dermatol Online J 17(2): 1.

2. Sayed FE, Dhaybi R, Ammoury A, Chababi M (2006) Angiolymphoid hyperplasia with eosinophilia: efficacy of isotretinoin? Head Face Med 2: 32.

3. Suzuki H, Hatamochi A, Horie M, Suzuki T, Yamazaki SA (2005) case of angiolymphoid hyperplasia with eosinophilia (ALHE) of the upper lip. J Dermatol 32(12): 991-995.

4. Park Y, Chung J, Cho CG (2002) Angiolymphoid hyperplasia with eosinophilia of the tongue: report of a case and review of the literature. Oral Oncol 38(1): 103-106.

5. Fernandes BF, Al-mujaini A, Petrogiannis-haliotis T, Al-kandari A, Arthurs B, et al. (2007) Epithelioid hemangioma (angiolymphoid hyperplasia with eosinophilia) of the orbit: a case report. J Med Case Reports 1: 30 .
6. Huang M, Lloyd WC, O'hara M (2008) Angiolymphoid hyperplasia with eosinophilia: an unusual presentation in a child. J AAPOS 12(3): 302-304.

7. Taylor SK, Meyerle JH, Glusac EJ (2016) Angiolymphoid hyperplasia with eosinophilia.

8. Wells GC, Whimster IW (1969) Subcutaneous angiolymphoid hyperplasia with eosinophilia. $\mathrm{Br} \mathrm{J}$ Dermatol 81(1): 1-14.

9. Rajendran R, Padmakumar SK, Kothawar S, Balaraman NM (2005) Angiolymphoid hyperplasia with eosinophilia (ALHE). J Oral Maxillofac Pathol 9(1): 24-26.

10. Mohammed AAM, Mohammed KF, Fares U, Karim L, Abdulla D (2011) Angiolymphoid hyperplasia with eosinophilia (epithelioid hemangioma) of the face: An unusual presentation. Int J Surg Case Rep 2(8): 258260.

11. Kuo TT, Shih LY, Chan HL (1988) Kimura Disease: Involvement of regional lymph nodes and distinction from angiolymphoid hyperplasia with eosinophilia. Am J Surg Pathol 12(11): 843-854.

12. Kung IT, Gibson JB, Bannatyne PM (1984) Kimura's Disease: a clinico-pathological study of 21 cases and its distinction from angiolymphoid hyperplasia with eosinophilia. Pathology 16(1): 39-44.

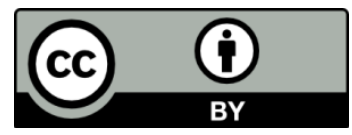

